\title{
Teenager with upper gastrointestinal obstruction - keep in mind the rare causes
}

\author{
Livia E. Brezeanu' ${ }^{1}$, Oana M. Guta', Raluca M. Vlad ${ }^{1,2}$, Anatolie Nidelcu', \\ Mirela Pavelescu, ${ }^{1,2}$, Daniela Pacurar1,2 \\ 1“Grigore Alexandrescu” Emergency Children's Hospital, Bucharest, Romania \\ 2"Carol Davila" University of Medicine and Pharmacy, Bucharest, Romania
}

\begin{abstract}
Introduction. Superior mesenteric artery syndrome is a rare cause of upper gastrointestinal tract obstruction. Case presentation. A 17-year-old adolescent is admitted in the Pediatrics Department of "Grigore Alexandrescu" Hospital in Bucharest in July 2018 for episodes of vomiting associated with epigastric pain. The patient started presenting persistent abdominal pain since five years before, apparently after an appendectomy. In the last year, he associated heartburn, dysgeusia, food vomiting and postprandial plenitude. A diagnosis of $\mathrm{H}$. pylori acute gastritis was initially set and he received triple therapy with temporary relief. Due to chronic digestive symptoms, the adolescent presented anxiety related to food intake with extremely reduced appetite, food selectivity and lack of weight gain. The clinical examination revealed marfanoid phenotype, low weight, chest deformity, excavated abdomen, epigastric pain and clapotage. Laboratory investigations were within normal limits. Esogastroduodenal barium examination showed an important gastric stasis with an elongated stomach, dilation of the second part of the duodenum with delayed distal passage. Abdominal ultrasound revealed reduced aorto-mesenteric distance at the third part of the duodenum. Upper gastrointestinal endoscopy found barium residue in the stomach 24 hours after the contrast study and a spastic pylorus, with impossible safe duodenal passage. Abdominal computed tomography confirmed obstruction of the third part of the duodenum by vascular compression at the aorto-mesenteric angle, a specific image for superior mesenteric artery syndrome. The patient underwent laparoscopic surgery with a good outcome.

Conclusions. Although a rare entity, duodenal obstruction by aorto-mesenteric clamp must be taken into account in the differential diagnosis of vomiting. An early radiologic diagnostic spares the patient of chronic digestive disease with negative impact on nutritional status and quality of life.
\end{abstract}

Keywords: vomiting, duodenal obstruction, marfanoid phenotype, aorto-mesenteric clamp

\section{INTRODUCTION}

Superior mesenteric artery syndrome (also known as: Wilkie syndrome/Cast syndrome/aorto-mesenteric clamp/chronic duodenal ileus) is a rare cause of upper gastrointestinal (GI) obstruction $(1,2)$. The incidence varies from $0.2 \%$ to $0.78 \%$, mostly in women, but has been also described in older children and adolescents. So far about 400 cases have been described in the literature $(3,4)$. The main pathophysiological feature of the syndrome is the reduction of angle between the superior mesenteric artery and abdominal aorta (less than 22 degrees) which leads to compression of the third portion of the duodeum or the left renal vein (5).

Clinical presentation varies from acute symptoms of duodenal obstruction or left varicocel to chronic GI symptoms such as: postprandial epigastric pain, early satiety or weight loss. The syndrome can be either congenital or acquired. The congenital form may be explained by a congenitally short ligament of Treitz. The main cause of reduction in the aorto-mesenteric angle in the acquired type is the decrease of perivascular fat that surrounds the vessels (6). Predisposing factors for the syndrome are: debilitating illnesses, malabsorbtion syndromes, corrective surgery for scoliosis and other disorders associated with extreme weight loss (7).

\section{CASE REPORT}

We present the case of a 17 years old adolescent, admitted in the Pediatrics Department of "Grigore Alexandrescu" Emergency Hospital for Children in Bu- 


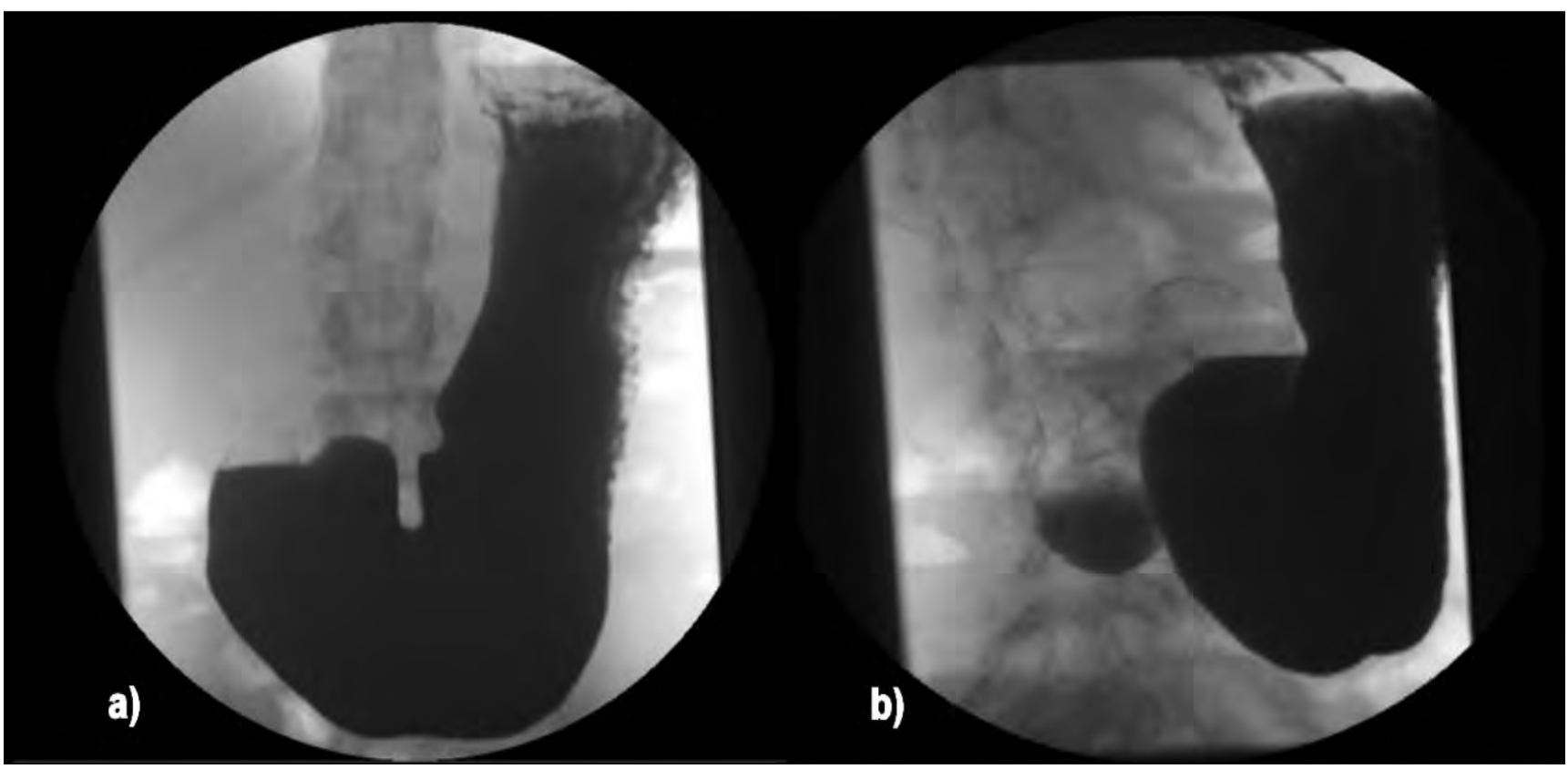

FIGURE 1. Esogastroduodenal barium study: (a) Elongated stomach, important gastric stasis (b) Dilation of the second part of the duodenum, delayed distal passage

charest in July 2018 for episodes of vomiting associated with epigastric pain, postprandial plenitude, pyrosis and dysgeusia. The past medical history of the patient was relevant for: an appendectomy for anatomic variant of retrohepatic appendix complicated with peritonitis five years prior to current admission, astigmatism diagnosed at the age of twelve and pectus excavatum complicated with respiratory insufficiency-restrictive type, surgically treated with metal plate orthopedic implant, extracted one month before admission. Regarding the family history, the patient's father was diagnosed with atrial septal defect and prolactinoma.

The adolescent started presenting intermittent abdominal pain five years before, apparently after the appendectomy. In the last year, he associated heartburn, dysgeusia, food vomiting and postprandial plenitude. A diagnosis of Helicobacter Pylori acute gastritis was initially set and he received triple therapy with temporary relief. Later on, the symptoms reappeared and in June 2018 an upper GI tract endoscopy was performed in an outpatient setting, showing gastric stasis with negative rapid urease test for Helicobacter Pylori. For the gastroesophageal reflux disease and esophageal motility disorder the patient was prescribed treatment with proton pomp inhibitors and prokinetics. Symptoms persisted despite medical treatment, so the patient was admitted for further investigations. It is important to highlight that due to chronic digestive symptoms, the teenager presented anxiety related to food intake with extremely reduced appetite, food selectivity and lack of weight gain.

Clinical examination revealed a thin teenager (weight for age under the 3 th percentile, body mass index for age under the 3th percentile) with normal stature (height for age on the 50th percentile), excavated abdomen, painful in the epigastric area, clapotage on palpation. Specific physical traits consistent with marfanoid phenotype were observed: kyphoscoliosis, pectus excavatum, joint hypermobility, dolicostenomelia and arachnodactyly. Ophthalmologic and cardiac examination were within normal limits.

Laboratory investigations were undertaken: complete blood count, liver and pancreatic enzymes, renal function, proteinemia, blood electrolytes, thyroid hormones and anti-transglutaminase antibodies were within normal range.

A diverse range of pathologies were taken into account for the differential diagnosis: gastroesophageal reflux, esophageal motility disorders, acute gastritis, gastric or duodenal ulcer, acute pancreatitis, neoplasic disease or causes of mechanical obstruction of the gastrointestinal tract.

In order to rule out mechanical obstruction, imaging studies were performed. Esogastroduodenal barium examination showed an important gastric stasis with elongated stomach with its inferior pole located distally to the iliac crest and dilation of the second part of the duodenum with delayed distal passage (Figure 1).

Abdominal ultrasound revealed reduction of aorto-mesenteric distance at the third part of the duodenum $(4 \mathrm{~mm})$, with anterograde dilation of the second part of the duodenum (43 mm) (Figure 2).

Furthermore, upper gastrointestinal endoscopy showed barium residue in the stomach 24 hours after 


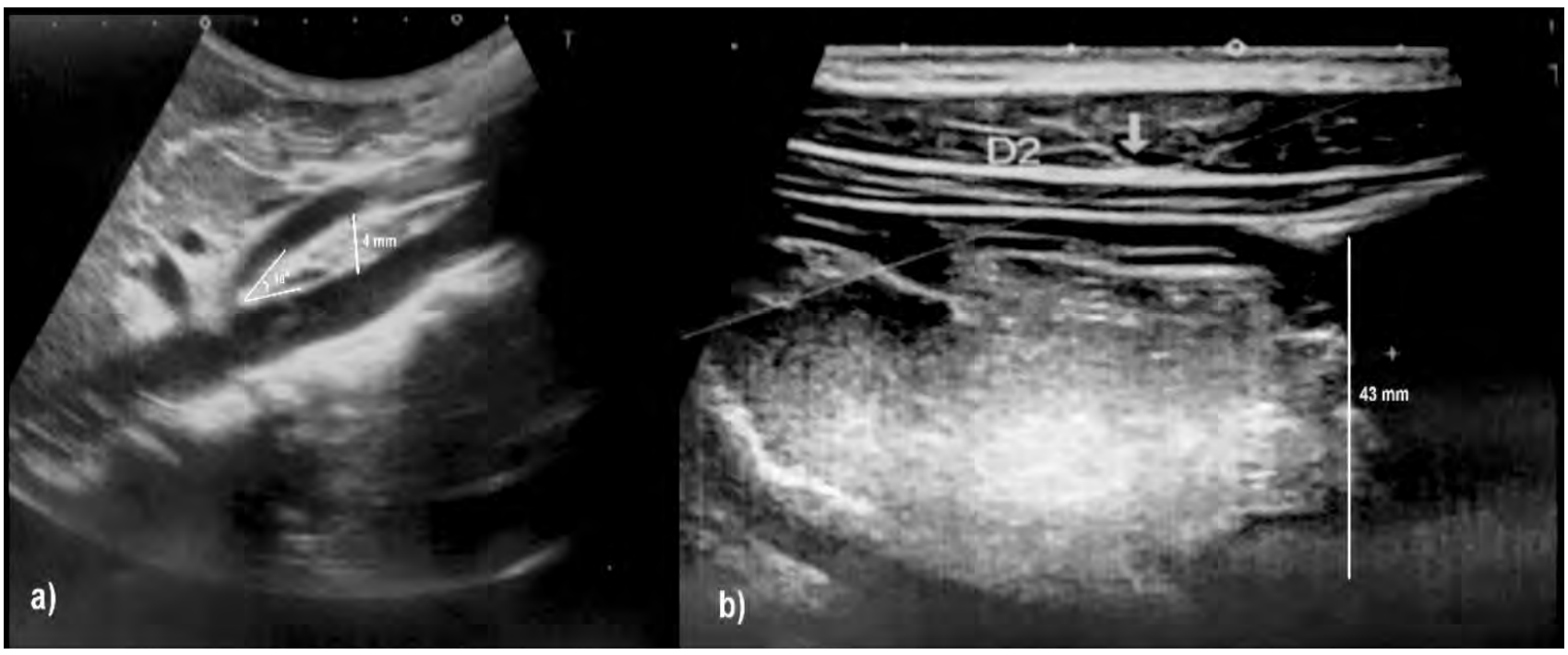

FIGURE 2. Abdominal ultrasonography: (a) Reduced aorto-mesenteric distance at the 3rd part of the duodenum (b) Dilation of the second part of the duodenum

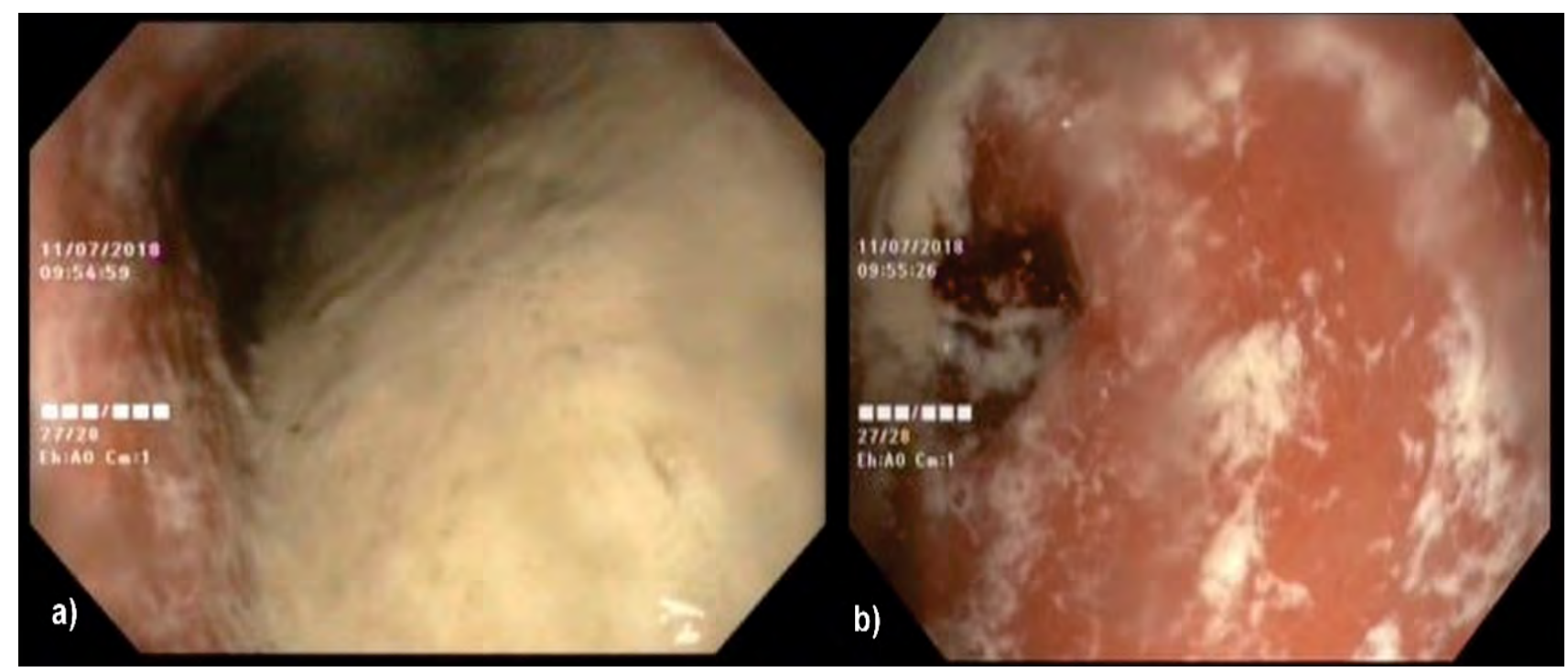

FIGURE 3. Upper GI tract endoscopy: (a) Barium residue in the stomach b) Spastic pylorus

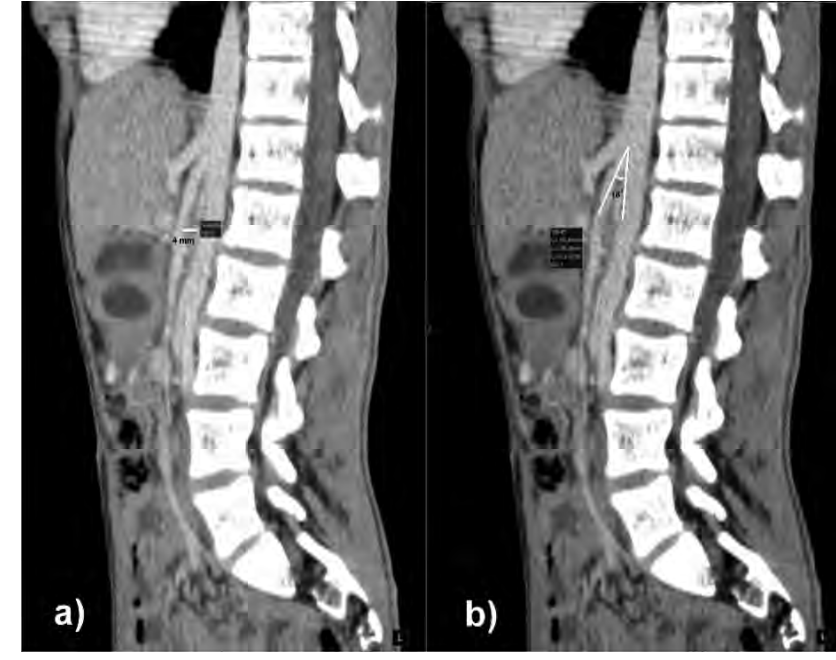

FIGURE 4. Abdominal contrast CT: (a) Aorto-mesenteric distance $=4 \mathrm{~mm}$ (b) Aorto-mesenteric angle $=18^{\circ}$

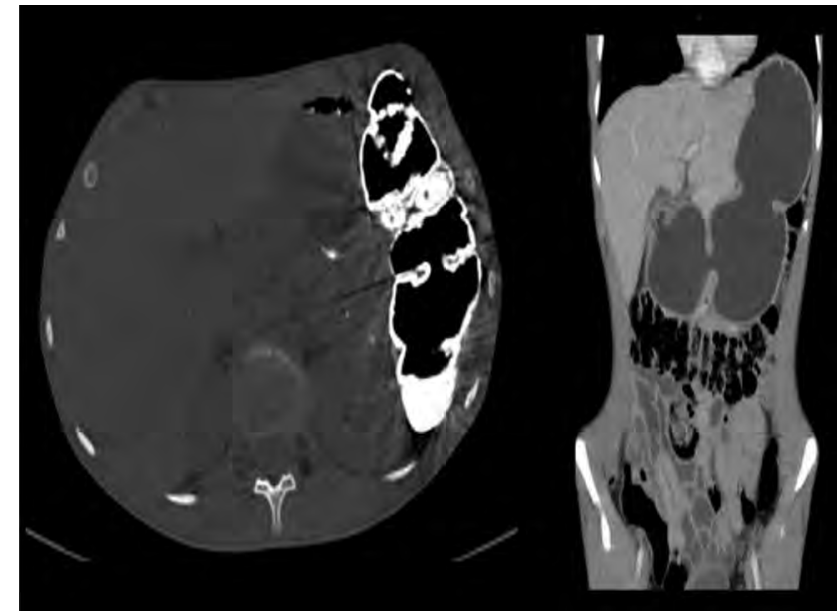

FIGURE 5. Abdominal contrast CT: Dilation of the stomach and duodenum 
the contrast study was performed with important gastric stasis and a spastic pylorus which did not allow the passage of the endoscopy tube in the duodenum (Figure 3).

Abdominal computed tomography (performed 3 days after the barium meal) confirmed the obstruction by vascular compression of the third part of the duodenum at the aorto-mesenteric angle: aorto-mesenteric distance was $4 \mathrm{~mm}$, aorto-mesenteric angle, 18 degrees (Figure 4), a specific finding for superior mesenteric artery syndrome. The stomach and second part of the duodenum were severely dilated (Figure 5).

Given the chronic symptoms of the patient associated with negative impact on nutritional status and life quality, surgical treatment was proposed by our pediatric surgery department. The parents initially refused the intervention. Later on, laparoscopic surgery with Roux en $Y$ intestinal derivation was performed in a private clinic, with favorable outcome (complete remission of symptoms and significant weight gain -10 $\mathrm{kg}$ in 6 months.

\section{DISCUSSION}

Aorto-mesenteric clamp is a rare entity, a small number of cases have been published to this day in the literature. It has been described in children, but pediatric published data is scarce. To our knowledge this is the first pediatric case communicated by a Romanian group.

In the case presented, several risk factors may have been asocciated with further development of the syndrome, in pathologic vicious cycle. Weight loss due to reccurent vomiting and progressive restriction of food intake might have lead to reduction of the perivascular mesenteric fat pad, consequently decreasing the distance between the mesenteric artery and abdominal aorta. The same mechanism has been described in patients with anorexia nervosa $(8,9)$, in those with hypercatabolic states - trauma (10), patients undergoing bariatric procedures for morbid obesity (11) or patients suffering from intestinal malabsorbion. There are cases of SMA syndrome described in patients with HIV in advanced stages of disease (7).

Another predisposing factor described in the literature, especially in adolescents, is rapid linear growth without compensatory weight gain (7). We consider this may be the case for our patient. Possible peritoneal adhesions developed after the appendectomy may have contributed to the progression of the mechanical obstruction. The thoracic wall deformities the teenager associated (kyphoscoliosis, pectum excavatum) and also the corrective orthopedic treatment he received have also been contributing factors and they are described as such in the literature (12).
Symptoms of Wilkie syndrome can be either episodic or persistent and consist mainly in nausea, reccurent vomiting and postprandial epigastric pain, features that have also been encountered in the medical history of the adolescent. In a retrospective study that followed the cases of SMA syndrome from 1985 to 2005 in Wisconsin Children's Hospital (22 patients included), 59\% presented abdominal pain, 50\% nausea and vomiting, $32 \%$ early satiety and $18 \%$ anorexia (12).

A careful examination and medical history must be performed in all pediatric patients with vomiting unresponsive to antiemetic medication, especially given the fact that leading causes of vomiting in childhood are infections, food poisoning, gastroesophageal reflux disease, gastritis, so that this rare diagnostic may easily be overseen (13). A clinical presentation similar to our patient's (heartburn, vomiting and postprandial plenitude, persistent abdominal pain) should raise red flags to any physician looking at a child with symptoms and failure to thrive.

Any patient with sugestive symptoms for superior mesenteric artery syndrome should undergo imagistic investigation using upper gastrointestinal series, multisliced CT, CT angiography, magnetic resonance angiography, conventional angiography, ultrasonography and endoscopy $(5,14)$.

Studies have shown that abdominal ultrasound has a tight correlation with computer tomography in measuring of the aorto-mesenteric angle and distance (3). In the case presented, the ecographic findings were highly suggestive and the diagnosis was confirmed using multisliced CT with intravenous contrast. In superior mesenteric artery syndrome, the third portion of duodenum is compressed between the superior mesenteric artery and the abdominal aorta. Under normal circumstances, the fat and lymphatic tissue provide a cushion between these structures, maintaining a normal angulation of 38 to 65 degrees and a normal aorto-mesenteric distance of 10 to $28 \mathrm{~mm}(7,15)$. The measured angle and aorto-mesenteric distance in the case presented were 18 degrees and $4 \mathrm{~mm}$ respectively, very low as compared to normal cited ranges.

Treatment options for Wilkie syndrome include conservative and surgical treatment. Conservative treatment consists of gastric decompression using nasogastric tube, correction of electrolyte abnormalities and nutritional support with either enteral feeding tubes or, in complicated cases, with total parenteral nutrition $(7,16)$. In case of failure of the conservative treatment, surgical treatment is recommended. Surgical options include: gastrojejunostomy, duodenojejunostomy or different types of intestinal derivative procedures. The approach can be either open surgery or 
laparoscopy (17). In one of the first studies published on SMA syndrome, describing 6 patients diagnosed after corrective surgery for spinal deformities, 5 received conservative treatment and only one case was treated by laparoscopic duodenojejunostomy (18). The patient had a very good postsurgical course with significant improvement of nutritional status and quality of life.

\section{REFERENCES}

1. Dorph MH. The cast syndrome; review of the literature and report of a case. N Engl J Med. 1950;243(12):440.

2. Wilkie DP. Chronic duodenal ileus. Br J Surg. 1921; 9:204.

3. Unal B, Aktaş A, Kemal G, Bilgili Y, Güliter S, Daphan C, Aydinuraz K. Superior mesenteric artery syndrome; CT and ultrasonography findings. Diagn Interv Radiol (2005)11(2):90-95.

4. Agrawal GA, Johnson PT, Fisherman EK. Multi detector row CT of superior mesenteric artery syndrome. J Clin Gastroenterol. 41 (2007) (1):62-65.

5. Welsch T, Buchler MW. Recalling superior mesenteric artery syndrome. Dig Surg. 24 (2007):149-156.

6. Farina R., Foti PV. Wilkie's syndrome. J Ultrasound. 2017, 20:339-34.

7. Scovell S, Hamdan A. Superior mesenteric artery syndrome, uptodate. com (Accesed on 24.06.2019)

8. Pentlow BD, Dent RG. Acute vascular compression of the duodenum in anorexia nervosa. Br J Surg. 1981;68(9):665.

9. Gwee K, Teh A, Huang C. Acute superior mesenteric artery syndrome and pancreatitis in anorexia nervosa. Australas Psychiatry. 2010; 18(6):523.

10. Smith BM, Zyromski NJ. Superior mesenteric artery syndrome: an underrecognized entity in the trauma population. J Trauma. 2008; 64(3):827.

11. Goitein D, Gagné DJ. Superior mesenteric artery syndrome after laparoscopic Roux-en-Y gastric bypass for morbid obesity. Obes Surg. 2004;14(7):1008.

\section{CONCLUSIONS}

Although a rare entity, duodenal obstruction by aorto-mesenteric clamp must be taken into account in the differential diagnosis of vomiting. An early radiologic diagnostic spares the patient of chronic digestive disease with negative impact on nutritional status and quality of life.

Conflict of interest: none declared Financial support: none declared

12. Biank $\mathrm{V}$, Werlin S. Superior mesenteric artery syndrome in children: a 20-year experience. J Pediatr Gastroenterol Nutr. 2006 May; 42(5):522-5.

13. McCollough M, Sharieff GQ. Abdominal pain in children. Pediatr Clin North Am. 2006;53:109e113.

14. Wee JW, Lee TH, Lee JS, Kim WJ. Superior Mesenteric Artery Syndrome Diagnosed with Linear Endoscopic Ultrasound in a Patient with Normal Body Mass Index. Clin Endosc. 2013 Jul;46(4):410-3.

15. Lalanne MA, Ocantos JA, Coronil JR, Gentile E, Cabrera MI, Sinclair ME, Seehaus AC, García-Mónaco RD. The aorto-mesenteric angle and its elements: Clinical and imaging features of vascular and gastrointestinal compression syndromes. Proceedings of the European Congress of Radiology (ECR). Vienna, Austria 2013 (poster C-1800).

16. Munns SW, Morrissy RT. Hyperalimentation for superior mesentericartery (cast) syndrome following correction of spinal deformity. J Bone Joint Surg Am. 1984;66(8):1175.

17. Gersin KS, Heniford BT. Laparoscopic duodenojejunostomy for treatment of superior mesenteric artery syndrome. JSLS. 1998; 2(3):281.

18. Sapkas G, O'Brien JP. Vascular compression of the duodenum (cast syndrome) associated with the treatment of spinal deformities. A report of six cases. Arch Orthop Trauma Surg. 1981;98(1):7. 